\title{
Treatment of Febrile Neutropenia in Pediatric Cancer Patients. A Clinical Evaluation of Effectiveness of Emperical Oral Antibiotic Therapy
}

\author{
${ }^{1}$ Dr. Rupa Rana Shahi M.D. ${ }^{2}$ Dr. Gu Shao Qin M.D. PhD. \\ ${ }^{1}$ Resident (Department of Pediatrics No. 1 people's Hospital, Zhenjiang, China), \\ ${ }^{2}$ (Head Department of Pediatrics No. 1 people's Hospital, Zhenjiang, China)
}

\begin{abstract}
:
Introduction: Febrile neutropenia in cancer patients represents a common complication from chemotherapy that is potentially lethal. However in patient with fever and neutropenia during cancer chemotherapy who have a low risk of complications, oral antibiotic may be an acceptable alternative to intravenous antibiotics.

Methods: We conducted a prospective hospital based study to the patients aged 2-14 years who had fever and neutropenia during cancer chemotherapy for both hematological and solid tumors. Only low risk patients i.e. neutropenia of less than seven days, who were hemodynamically stable and ANC $>250 / \mathrm{mm}^{3}$ were included in the study. All the patients followed Guidelines for pre-treatment assessments and investigations before they were hospitalized and given oral antibiotic ofloxacin and were closely observed until fever subsided for more than 48 hours and improved from neutropenia. Results: A total of 92 cases were enrolled in the study. Out of 92 patients 1 patient was lost to follow-up, 14 needed IV antibiotics for different reasons and 74 patients (80\%) improved well with oral antibiotic only. The events that led to changing the treatment regimen were: grade III/IV vomiting (two cases), fever lasting for more than 72 hours (seven episodes), severe mucositis (one episode), anal cellulitis (one episode) and no obvious cause (three episodes). Conclusion: In hospitalized low risk patients who have fever and neutropenia, empirical therapy with oral ofloxacin may be a safe and effective alternative to IV antibiotics. Further study should be aimed at clarifying the definition of low risk pediatric patients.
\end{abstract}

Key words: Pediatric Febrile Neutropenia, Cancer, ANC.

\section{Introduction}

Advances in cancer chemotherapy have led to improved prognosis and treatment of pediatric malignancies. Bone marrow transplantation (BMT) is a curative strategy for treating various hematological disorders, including acute and chronic leukemia. But intensive chemotherapy and radiation can profoundly suppress immunity and patients have an increased susceptibility to infectious complications which may be fatal. Infections occur when the patient becomes neutropenic by intensive chemotherapy for treatment of malignancies or myelosuppressive therapy. Other contributory factors include the use of broad-spectrum antibiotics, corticosteroids and the use of venous catheters. Bacterial infections are still difficult to diagnose. This is because the symptoms are non-specific and can be confused with fungal and viral infection. However, bacterial infections are now well-treated and prevented by broad-spectrum antibiotics.

Due to the difficulty in diagnosis and its high morbidity and mortality, clinicians can start early empiric therapy in order to obtain a satisfactory outcome. Therefore, the general clinical practice is that patients with prolonged neutropenic fever first receive broad-spectrum antibiotic therapy. If a patient does not respond to antibiotic therapy, clinicians may then suspect the patient is suffering from fungal infection and switch to empiric antifungal therapy as early as possible

Until some years ago, the recommended treatment for febrile neutropenia was empiric broad spectrum intravenous antibiotics and hospitalization for all patients. Among febrile neutropenic patients only a small proportion is at risk of serious complications and death,

The definition of fever for the pediatric oncology patient, it is most often defined as a single oral or equivalent temperature of greater than 38.3_C (101_F) or two consecutive temperatures greater than 38.0_C (100_F) in a 12-hour period lasting at least 1 hour. Neutropenia was defined as a neutrophil count (ANC) of less than $1,000 / \mathrm{mcL}$, Neutrophil counts may vary on race and age. Neutropenia may be characterized as mild with an ANC of $1000-1500 / \mathrm{mcl}$, moderate $500-1000 / \mathrm{mcl}$ and severe $<500 / \mathrm{mcl}$. There are various causes of neutropenia. For example:

I. Infectious causes.

a. Viral infection.

b. Bacterial infection- Typhoid fever. 
c. Fungal infecton.

d. Protozoal infection.

ii. Drug Induced- Sulfa drugs, Chloramphemicol, Anticonvulsants, Phenothiazines etc.

iii.Cancer Chemotherapy and Radiotherapy .

iv. Immune Mediated. Developmental immaturity of innate immunity.

v. Hypersplenism.

vi.Bone Marrow infiltration - Leukemia.

viiBone Marrow Failure- Hypoplastic Anemia.

viiiNutritional Deficiency: Vit B12, Folate Deficiency.

Febrile neutropenia is one of the commonest complications of radiotherapy and chemotherapy. The immune system of children with malignancies is compromised by the anticancer drug and by the direct effect of cancer itself. In addition, frequent insertion of IV cannulas, indwelling catheter, malnutrition, prolonged exposure to antibiotics and frequent hospitalizations all add to risk for infection in these children. Both gram positive and gram negative infections, anaerobic infection, fungal infections may occur in these patients. The lack of neutrophils can lead to a loss of inflammatory response; hence fever may be the only manifestation of infection. So in neutropenic patients, only fever warrants the use of empirical antibiotics which cover both Gram positive and Gram negative pathogens. Before starting antibiotics we should do thorough physical examination in these patients and investigations like $\mathrm{CBC}$, blood culture, urine culture, X-ray chest and swab culture from different sites of body. If culture positive and sensitivity reports are available, we have to give the antibiotic accordingly. In developed countries, about $75 \%$ of children with fever and neutropenia were ultimately found to have a documented site of infection ${ }^{23}$ In most of the centers, the patient with febrile neutropenia is treated with IV antibiotics, usually with third generation cephalosporin plus aminoglycosides. The first line antibiotic therapy should take into consideration according to the type of microbe anticipated and local resistance patterns encountered at each institution4. In addition antibiotic choice may be affected by cost, drug allergy, drug availability, renal function etc. The empirical use of oral antibiotics has been shown to be safe in low risk adults who have no evidence of bacterial focus or signs of significant illness like rigor, hypotension, and change in mental status, however substantive data for this approach is lacking in children. There are few reports that were found regarding oral antibiotic therapy to low risk group of pediatric patient with febrile neutropenia. Alison Freifeld et.al. ${ }^{5}$ concluded that oral ciprofloxacin and amoxy-clav is safe and effective for patients with low risk febrile neutropenia. Winfried VK et al $\mathbf{6}$ also reported that in low risk partients with febrile neutropenia, oral therapy with ciprofloxacin plus amoxy-clav is as effective as intravenous therapy. If we could treat patients with oral antibiotics without compromising the outcome would be advantageous because oral antibiotics are cheaper, and patients would not need intravenous cannulation; hence there would be lesser chances for hospital acquired infections. Aim of this research was to study the effectiveness of oral antibiotic (ofloxacin) therapy to low risk febrile neutropenic patients.

\section{Material And Methods}

The study was carried out in Pediatric Oncology Ward of No.1 People's Hospital, from February 2012 to November 2012 i.e. duration of ten months. In a single institution, a hospital based prospective study, we followed 92 episodes of febrile neutropenia . Only low risk patients i.e. neutropenia of less than seven days, ANC $>250 / \mathrm{mcl}$, without any signs of shock were included in the study. All the patients after they went through pre-treatment assessments and investigations were hospitalized and given oral antibiotic ofloxacin and were closely observed until fever subsided for more than 48 hours and improved from neutropenia. Those patients predicted at low risk for complications, who were not receiving antibacterials at fever onset and were eligible for treatment with oral antibiotics, were treated with ofloxacin and were discharged if they were clinically stable or improving after an initial observation period. The primary end point of the study was the rate of resolution of the febrile neutropenic episode without complications, among these early discharged patients.

\section{Risk factors used to exclude patients from the low-risk protocol were:}

- Age $<1$ year

- hemorrhage

- Dehydration

- Metabolic instability

- Altered mental or neurological status

- Pneumonitis

- Mucositis

- Unable to tolerate oral fluids or having symptoms necessitating iv supportive therapy.

- Shock or compensated shock 
- respiratory distress/compromise

- $\quad$ perirectal or other soft tissue abscess,

- rigors

- $\quad$ irritability/meningism, or

- organ failure

- Acute lymphoblastic leukemia (ALL) at diagnosis/relapse <28 days, ALL not in remission (>28 days), acute myeloid leukemia (AML), infant ALL, intensive B-NHL (non-Hodgkin lymphoma) protocols, hematopoietic stem cell transplant, or sequential high dose chemotherapy with peripheral blood stem cell rescue.

- Intensive care admission during last FN episode, non adherence (for example social concerns), or inability to tolerate oral antibiotics.

- Positive blood culture result at 48 hours, ANC $<100 / \mathrm{mcl}$ at 48 hours, or child not clinically well at 48 hours (clinical judgment).

- Whereas this detailed list of risk factors may serve as an important decision aid for early discharge strategies, it cannot be considered as a validated risk prediction tool for initial risk stratification in pediatric patients.

- Neutropenia expected to last more than 7 days.

- Haemodynamically unstable.

- Inadequate hepatic and renal function.

- $\quad$ Patients with abdominal pain, nausia, vomiting or diarrhea.

- Intravascular catheter infection.

- Patients those who received antibiotics within the last 72 hrs or 7 days.

- Patients with new pulmonary infiltrates or respiratory insufficiency.

- Patients who had received autologous stem cell transplants.

\section{Study Design}

A history was obtained and physical examination was performed on each patient within an hour after admission. A complete blood count, chest x-ray, routine examination of urine, blood culture, urine culture, swab cultures; if indicated, were performed before empirical therapy was begun. After the initial evaluation, patients were enrolled in the study according to above inclusion criteria (low risk group). They were treated with oral antibiotic ofloxacin. High risk patients who had ANC $<250$ were not included in this study and were treated with IV. antibiotics. Patients were treated until neutropenia resolved (ANC >1000) and fever subsided. The relatives or the care taker of the patients were counseled about the treatment protocol and verbal consent was also taken.

\section{Clinical Assessment}

All the patients were closely monitored by doing a thorough clinical assessment and laboratory investigations. Changes in treatment regimen into IV antibiotics were made if the patient did not improve (For example; positive blood cultures showing resistance to the initiated therapy, persistent high fever, persistent vomiting, decreasing pattern of ANC and signs of shock present). These patients were regarded as treatment failures and IV antibiotics either cefepime monotherapy or ceftazidime and amikain with or without addition of vancomycin and antifungals were given depending on the severity and clinical judgement of condition.

\section{Evaluation of Response of Therapy}

Clinical outcomes were prospectively defined. Oral antibiotic therapy was considered to have been successful if the patient improved without any change of antibiotic and increased ANC. Treatment was considered to have failed if the regimen had to be modified by addition of one or more antibacterial or antifungal agents. All the patients were followed up to seven days. Statistical Analysis was done in terms of percentage effectiveness of therapy.

\section{Results}

From February 2012 to November2012, a total of 92 patients were enrolled in this study. Out of these, $77(83 \%)$ patients improved only with oral antibiotic and $14(15 \%)$ patients needed IV antibiotics for various reasons as stated above Oral antifungal was added to 5 patients as they developed mucositis. No mortality was noted in these studied patients. One patient was lost to follow up and could not be traced 


\section{Discussion}

Fever occurring in neutropenic patients still remains a challenging and common life-threatening complication of cancer chemotherapy. The common practice is to admit the patient to hospital and treat empirically with broad-spectrum intravenous antibiotics with or without addition of an antifungal.

Although many guidelines have been developed to assist care providers to risk-stratify their patients and select initial therapies, it has become obvious that patients experiencing febrile neutropenia induced by chemotherapy do not constitute a homogeneous group ${ }^{13}$

We often treat febrile neutropenia with IV amikacin plus cefepime. We found that oral ofloxacin is an effective oral therapy to the patients with febrile neutropenia of a low risk category. Treatment was successful in $>80 \%$ of all episodes with oral therapy. Our definition of low risk group was taken as explained before in patients with history of fever of less than three days and those that had no features of shock, abdominal tenderness or diarrhea. The case for selection of low risk patient is extremely important. All patients with high risk febrile neutropenia must be treated with IV. antibiotics without any delay. In this study we gave IV antibiotics if ANC $<250 / \mathrm{cmL}$. But in some studies it was found that even an ANC $<100 / \mathrm{cml}$ were successfully treated with oral antibiotics. Alison Freifeld, Donna Marchigiani et al reported that $71 \%$ of patients who were given oral antibiotic group having a mean ANC of $81 / \mathrm{cml}$ were successfully treated with oral ciprofloxacin plus amoxy-clav7. Alejandria MM, Panaligan MM, Molina FG et.al., during the 8th International Cochrane Colloquium 2000, cape town, also reviewed the data regarding oral versus intravenous antibiotic for low risk febrile neutropenia and concluded that oral antibiotic regimens were equally effective as standard intravenous antibiotic therapy for the treatment of patient with low risk febrile neutropenia.8 Similarly, Winfried V. Kern, Alain Cometta and et. al. reported that $86 \%$ of their patients were successfully treated with oral antibiotics 9 . Jean Klastersky, Marianne Paesmans et al studied 383 first febrile neutropenic episodes predicted at low risk of complication, among these, 178 patients, mainly with solid tumors were treated orally; Seventy-nine patients (44\%) were discharged early (with a median time to discharge of 26 hours); no complications occurred among them except that three patients had to be readmitted, resulting in a success rate of $96 \%$ (95\% CI, 92\% to 100\%).10 Liat Vidal, Mical Paul, Itsik Ben doret al, in their review found out that there was no significant difference in mortality rate comparing oral and IV antibiotic treatment. Moreover, treatment failure rates were also similar. No significant heterogeneity was shown for the primary outcomes. This effect was stable in a wide range of patients. Quinolones alone or combined with other antibiotics were used with comparable results. Adverse reactions, mostly gastrointestinal, were more common with oral antibiotics.11,12.

\section{Limitation}

This is a small study, we need to do a bigger study to verify whether or not to use oral antibiotic in febrile neutropanic patients. The study was done in only those patients who were considered as low risk. We had not included those patients with an ANC of less than 250/ cmm. In some studies it was found that patients with

ANC $<100 / \mathrm{cml}$ were also treated safely with oral antibiotics.

\section{Conclusion}

Though we are treating the patient with febrile neutropenia with IV antibiotics as first line therapy, the larger study comparing the efficacy of the use of oral antibiotic should be done. If we could treat those patients with febrile neutropenia with oral antibiotic, it would be more cost effective and would also decrease their hospital stay. We conclude that in our study we could successfully treat low risk febrile neutropenic patients with oral antibiotic. In low-risk hospitalized patients with cancer who have fever and neutropenia, empirical oral-therapy with ofloxacin was found to be as effective as intravenous-therapy. However, how to use these regimens appropriately in individual patients remains challenging. Further studies may be necessary before adopting this oral regimen for outpatient use.

\section{References}

[1]. Behrman, Kliengman and Arvin, Neutropenia,Nelson Textbook of Paediatrics 17th Ed Saunders Elsevier p.717-20.

[2]. Agrawal BR, Perilongo G, Rogers P, Strahlendorf C,Eden OB. Practical Paed Oncology.International Society of Paediatric Oncology (SIOP),2nd Ed, 29-31.

[3]. Behrman, Kliengman and Arvin, Febrile Neutropenia, Nelson Textbook of Paediatrics, $17^{\text {th }}$ Ed,Saunders Elsevier p.853-855.

[4]. Bob Phillips, Karen Selwood Sneila M Lane et al.Variation in Policies for the Management of Febrile Neutropenia in United Kingdom Children's CancerStudy Group Arch. Dis. in Children 2007,92,495-98.

[5]. Antibacterial Prophylaxis in Patients with Cancer and Neutropenia. N Eng J Med 2006 Jan 5;354(1):90-4.

[6]. Kern WV, Cometta A, Bock Rd, Langenaeken J et al, Oral versus Intravenous Empirical Antimicrobial Therapy for Fever in Patients with Granulocytopenia who Are Receiving Cancer Chemotherapy. N Eng J Med 1999 Jul 5;341:312- 318. 
[7]. Alison Freifeld et al double blind comparison of empirical oral and intravenous antibiotic therapy for low risk febrile patients with neutropenia during cancer therapy. N Eng. J of Med,1999 341,305-311.

[8]. M.M. Alejandria, M.M. Panaligan, F.G. Molina, M.F.P. Raymundo.Colloquim Abstracts. $8^{\text {th }}$ International Cochrane Colloquium. 2000, Cape Town

[9]. Winfried V Kern, Alain et. al. Oral vs IV empirical antimicrobial therapy for fever in patient with granulocytopenia who are receiving chemotherapy.N Eng J Med, 1999,Vol.341,312-318.

[10]. Klastersky J, Paesmans M. et al. Outpatient Oral Antibiotics for Febrile Neutropenic Cancer Patients Using a Score Predictive for Complications. Journal of Clinical Oncology, 2006; 24:25,4129- 4134.

[11]. Vidal L, Paul M,Bendor I et al. Oral versus intravenous antibiotic treatment for febrile neutropenia in cancer patients: a systematic review and meta-analysis of randomized trials. Journal of Antimicrobial Chemotherapy 2004 54(1):29-37.

[12]. Vidal L, Paul M, Ben-dor I. et,al. Oral vs IV Antibiotic Treatment for Febrile Neutropenia in Cancer Patients. Cochrane Database, 2004, Issue 4, Art. No: CD 003992.

[13]. Talcott JA, Whalen A, Clark J et al. Home antibiotic therapy for low-risk cancer patients with fever and neutropenia: a pilot study of 30 patients based on a validated prediction rule. J Clin Oncol 1994;12(1):107-14.

[14]. Vidal L, Ben dor I, Paul M, Eliakim-Raz N, Pokroy E, Soares-Weiser K, Leibovici L. Oral versus intravenous antibiotic treatment for febrile neutropenia in cancer patients. Cochrane Database of Systematic Reviews 2013, Issue 10. Art. No.: CD003992.DOI: 10.1002/14651858.CD003992.pub3.

[15]. Ângela Rech Cagol'; Cláudio Galvão de Castro Junior ${ }^{\mathrm{II}}$; Maria Cristina Martins ${ }^{\mathrm{III}}$ Oral vs. intravenous empirical antimicrobial therapy in febrile neutropenic patients receiving childhood cancer chemotherapy J. Pediatr. (Rio J.) vol.85 no.6 Porto Alegre Nov./Dec. 2009 